\title{
Research of Reliability Enhancement Testing about Wear Failure Component Based on Virtual Prototype Technology
}

\author{
Zhao Jin ${ }^{1, a^{*}}$, Fei Gao ${ }^{1, b}$, Hejia Li ${ }^{1, c}$ and Jiuchao $\mathrm{Li}^{1, \mathrm{~d}}$ \\ ${ }^{1}$ No.1, Huayuan Road, Changchun City, Jilin Prov. China 130117 \\ a781693564@qq.com, bgaofei_1201@126.com, ${ }^{\mathrm{c}, \mathrm{d} 247658766 @ q q . c o m}$
}

\section{Keywords: Virtual Prototyping; Simulation; RET; Wear; RET-CAE Technology}

\begin{abstract}
Aiming at the problems of long life cycle and high cost of mechanical component wear life test, this paper proposes a virtual prototyping simulation technology to the study of wear failure Reliability Enhancement Testing (RET). Based on the analysis of wear failure, the Enhancement testing mechanism based on wear failure is proposed, the wear failure test technology principle based on virtual prototype simulation technology of RET is determined, and the Enhancement Coefficient is deduced. Taking a feeding mechanism as the research object, the virtual prototype model was established, and the study of RET about wear life of the pin and pin hole was simulated. The results show that the adopted technical scheme is feasible and provides a useful reference for the application of virtual prototype technology in the field of RET.
\end{abstract}

\section{Introduction}

RET is a new type of accelerated stress test method for the purpose of stimulating faults. Rapid testing can be performed in a short period of time by applying a test that is far more stressful than the specification [1].

With the wide application of CAE technology, virtual prototype simulation test has become an important trend in the development of Reliability Testing technology [2]. Combining the virtual prototype simulation technology with RET to build a virtual RET platform environment, and based on this, research on the virtual RET technology, the use of computer simulation to realize RET technology idea, will gradually develop into Efficient reliability growth technology.

This paper takes the wear failure mode in the mechanical system as the research object, explores its RET theory and technical methods, and simulates the typical pin and pin hole clearance wear components as an example. Provides reference for further research and application of mechanical system RET.

\section{Theoretical Basis of RET about Wear Failure}

Wear mainly occurs on the surface of two interacting components in a mechanical system. When the material wears to the extent that the component profile is not sufficient to transmit motion or power or transmit motion, and the power is not reliable, it can be considered that the wear causes the end of the component life. RET achieves the goal of inducing the failure of the test object in a short time by increasing the stress level of the test work, thereby rapidly evaluating the reliability of the test object. Therefore, in the wear-enhancement testing, the mechanism of the wear-failure-enhancement testing must be clarified first, and then the technical approach to improve the stress level is determined for the analysis of specific parts, and then the simulation test of the enhanced test is performed through the virtual prototype.

Mechanism of RET about Wear Failure. The test mechanism is the theoretical basis of RET, and it is of great significance to the in-depth research and application of RET. [3, 4]

The amount of wear $\mathrm{W}$ depends mainly on the surface pressure $\mathrm{p}$, the sliding speed $\mathrm{v}$, the acting time t, [5, 6] namely:

$$
W=K_{\mathrm{W}} p^{m} v^{n} t
$$


In this equation: $\mathrm{W}$ is the amount of wear, that is, the amount of wear per unit area of the contact area; $K_{W}$ is the conditional condition coefficient, related to the factors such as the material, the surface quality and the lubrication condition; $p 、 v$ is the (surface) positive pressure, the relative sliding speed; $m 、 n$ is the pressure and the index of the effect of sliding speed on the amount of wear; $t$ is the wear time. Among them, $K_{W} 、 m$ and $n$ are usually obtained through experimental fitting.

According to Eq. 1, the positive pressure and relative velocity between the contact specimens are the main factors that determine the amount of wear when the specimen material is determined. Therefore, the primary task of RET about wear failure is to specifically analyze the working principle of test object. The working principle is that, based on the stress and relative motion of the components, a feasible RET scheme is formed by increasing the surface positive pressure or relative motion speed between the components.

Technical Principles of RET about Wear Failure. Under normal circumstances, the basic requirement of RET is to establish an enhanced stress limit, especially virtual RET. Therefore, this paper puts forward the principle of wear virtual reinforcement test technology to determine the following principles:

First, objective conditions can be achieved. This means that the applied strengthening stress should be within the objective condition energy level.

Second, cannot change the failure mechanism. The applied strengthening stress level cannot change the failure mechanism of the test piece. The main reason for the deviation of the failure mechanism in the wear failure RET is that the strength of the component under stress is not enough, and the component fails in the form of a fracture. And this cannot be observed in the virtual test. Therefore, it is necessary to check the strength of the parts that may have a failure due to enhanced stress. The wear failure RET mainly accelerates the failure of parts by changing the strength of the parts.

\section{Derivation of Enhancement Coefficient about Wear}

In order to compare the effect of different stress levels, the concept of Enhancement Coefficient was introduced. That is, comparing the test results under the enhanced stress level with the results under normal stress levels can more directly reflect the degree of enhancement. For different failure modes, the content of Enhancement Coefficient's calculation is different. RET of mechanical wear failure, after the part wears, its external shape changes, the surface appears the furrow or the pit and so on the wear form, at the same time along with the wear debris generation. Therefore, the amount of mechanical wear at different stress levels can be used as a measure of the effect of enhancement. In order to measure the relationship between the degree of wear of the mechanism after completing the action under different stress levels, the effect of the test is characterized by the amount of wear after a single action of the component. Enhancement coefficient wear enhancement coefficient $\mathrm{K}_{\mathrm{e}}$ can be expressed as:

$$
K_{e}=\frac{H^{\prime}}{H}
$$

In this equation: $H$ and $H^{\prime}$ denote the single wear amount of the parts working under normal working conditions and enhancement working conditions, respectively.

Since the load spectrum and the velocity spectrum of the simulation test are constantly changing with time, a segmentation approximation method is adopted to facilitate the calculation: Using the simulation sampling interval as the time cell $\Delta \mathrm{t}$, the contact pressure and the relative velocity are handled as constants at the micro cell $\Delta \mathrm{t}$.

For normal stress levels, let "a" be the number of sampling points of the load spectrum during the interaction of two parts. According to formula (1), the amount of wear on the component contact area $\mathrm{S}(\mathrm{i})$ within the time microelement $\Delta \mathrm{t}(\mathrm{i})$ can be expressed as: 


$$
W(i)=K_{W} p(i)^{m} v(i)^{n} S(i) \Delta t(i)
$$

In the Equations: $(\mathrm{i}=1,2,3, \ldots, \mathrm{a})$.

The total amount of wear of a single action of a component is:

$$
H=\sum_{i=1}^{a} W(i)
$$

For enhanced stress levels, the contact pressure and relative speed are set to $p^{\prime}$ and $v^{\prime}$, then the wear rate is:

$$
W^{\prime}(i)=K_{W} p^{\prime m} v^{\prime n}
$$

Let "b" be the number of sampling points of the load spectrum, then the amount of wear on the component contact area $\mathrm{S}^{\prime}(\mathrm{i})$ in $\Delta \mathrm{t}^{\prime}$ can be expressed as:

$$
W^{\prime}(i)=K_{W} p^{\prime}(i)^{m} v^{\prime}(i)^{n} S^{\prime}(i) \Delta t^{\prime}(i)
$$

The total amount of wear of a single action of a component is:

$$
H^{\prime}=\sum_{i=1}^{b} W^{\prime}(i)
$$

According to the above equations, we can get the enhancement coefficient about wear after single-action is:

$$
K_{e}=\frac{H^{\prime}}{H}=\frac{\sum_{i=1}^{b} W^{\prime}(i)}{\sum_{i=1}^{a} W(i)}=\frac{\sum_{i=1}^{b}\left[p^{\prime}(i)^{m} v^{\prime}(i)^{n} S^{\prime}(i) \Delta t^{\prime}(i)\right]}{\sum_{i=1}^{a}\left[p(i)^{m} v(i)^{n} S(i) \Delta t(i)\right]}
$$

According to the formula, the amount of wear can be calculated using computer programming, and then the enhancement coefficient under different working conditions can be obtained.

\section{Simulation of RET about Wear}

A feeding mechanism adopts a reciprocating push chain structure. It is an open chain drive system that is not connected end to end. And it is mainly used for straight-line pushing. This kind of feeding mechanism frequently starts and stops during work. And in its working process, the interaction between the pin hole and the pin shaft of the link plate is long, the load is large, and the relative sliding is frequent, which is a typical wear-out failure component. Its structure is shown in Fig. 1.

Working Principle. In the feeding mechanism, the force that the chain is subjected to before and after meshing with the sprocket is different. When the chain is in the chain box, it is pulled. But by the time the chain comes out, it is subjected to pushing pressure. This push-type chain drive essentially completes the transmission of force and movement through collisions. Due to the specificity of its power transmission, in order to accurately simulate its dynamic characteristics, using physical impact to study the contact between the chain roller and the sprocket, the chain roller and the box separator can more accurately reflect its dynamic characteristics.

Failure Mechanism. Chain wear is a common failure in chain drives. As an open chain transmission feeding mechanism, when the chain extends out of the chain box, the two chain plates are attached to one another to form a rigid body. In use, wear occurs due to friction, mainly occurs between the pin axis and the inner and outer link pin holes, resulting in loose fit, chain pitch larger, the chain positive bending string height difference, the effective extension of the chain length becomes shorter, causing the push Both the distance and the pushing speed have decreased, resulting in inaccurate movement of the mechanism. When the wear is serious, chain jam may occur 
and mechanical accidents may occur. As is shown in Fig. 2.

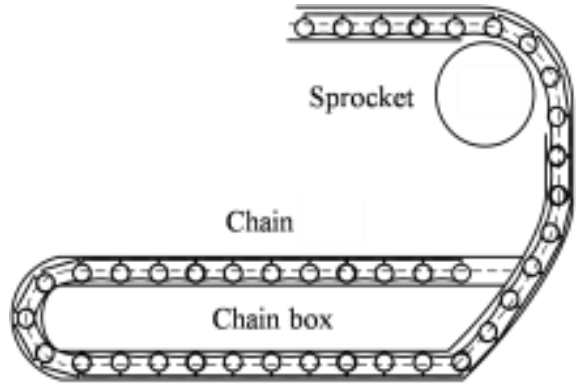

Figure 1. Schematic diagram of the chain

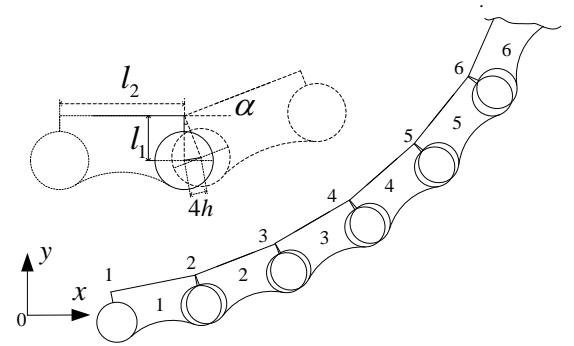

Figure 2. Wear-out failure model of chain

Technical Solutions of RET. From Equation (1), it can be seen that the generalized stress affecting the life has two pressures: $p$ and $v$.After simulation comparison, it was found that using $p$ is better than using $v$. However, in practice, adopting $v$ is easier to implement and closer to the actual situation. Therefore, it is more practical to study using $v$ to enhance the stress, so this paper uses the relative sliding speed to analyze the stress.

Simulation of RET. During the operation of the feeding mechanism, the force between the pin shaft and the pin hole of the chain plate and the relative movement speed are difficult to measure through the test. This article establishes the virtual prototype model of the feeding mechanism. Its structure and main components are shown in Fig. 3. Through simulation calculation, the collision load spectrum and relative angular velocity spectrum between the pin shaft and the pin hole can be easily obtained. It is successfully solved that in engineering practice, the collision load and relative rotation speed of the pin shaft and the pin hole are difficult to measure. The problem. By simply processing the relative angular velocity spectrum, a sliding velocity spectrum between the pin axis and the pin hole can be obtained.

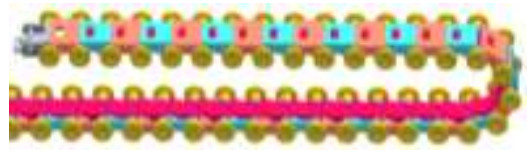

(a) Chain assembly

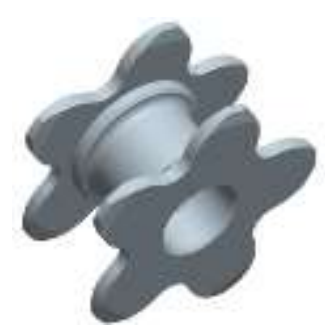

(c) Sprocket

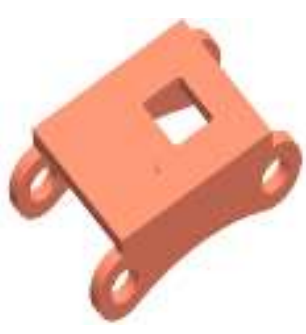

(d) Outer chain plate

Figure 3. Model of some components

Fig. 4 and Fig. 5 are the collision load spectrum and relative velocity spectrum between the pin and the pin hole under the normal operating conditions.

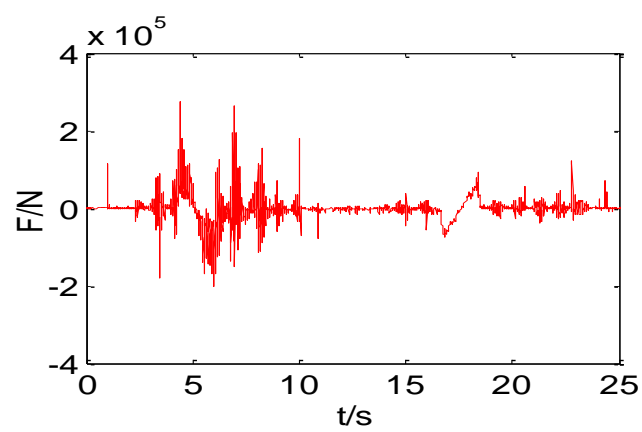

Figure 4. Load spectrum of the pin

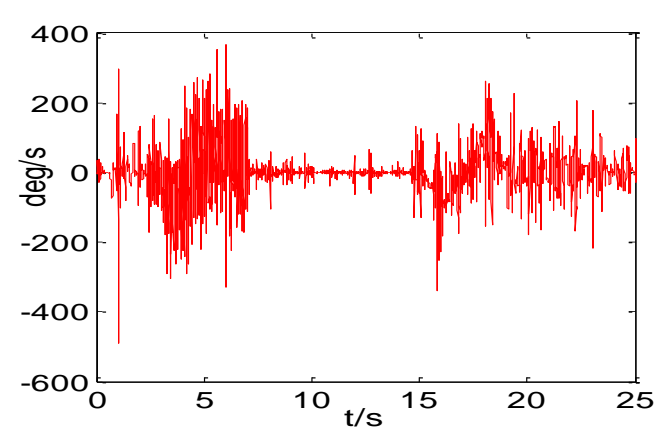

Figure 5. Relative velocity spectrum 
Analyzing two spectral lines, we will find that in the time periods $\mathrm{t}=3.8 \mathrm{~s} \sim 6.7 \mathrm{~s}$ and $\mathrm{t}=16.4 \mathrm{~s} \sim$ $19.3 \mathrm{~s}$, the amplitudes of the two lines are significantly increased, and the direction of the curve is obvious. This is because during these two periods of time, the pin shaft enters the meshing stage with the sprocket, the relative rotational speed and load will increase significantly, and the movement of the pin shaft will transition from the original linear motion to the rotation of the sprocket. Among them, $3.8 \mathrm{~s} \sim 6.7 \mathrm{~s}$ is the engagement phase between the pin and the driven sprocket, $16.4 \mathrm{~s} \sim 19.3 \mathrm{~s}$ is the engagement phase with the driving wheel. When the pin shaft is meshed with the driven wheel, the pin shaft is far away from the power source, so it meshes with the driven sprocket. When the vibration is more obvious than when engaging with the driving wheel, the spectrum line is in line with the actual situation. Both curves have obvious "burrs", which are caused by vibrations and impacts caused by the polygonal effect of the sprocket and the friction during the operation of the mechanism.

The load spectrum and relative velocity spectrum are obtained and the wear life can be calculated programmatically. Assuming that the allowable wear amount is 1 , Table 1 is the life data calculated under normal operating conditions and several enhanced working conditions.

We found that in this example, the logarithm of life is more convenient for data processing and curve fitting, so the table takes the usual logarithmic treatment of life.

Table 1 RET statistics

\begin{tabular}{|c|c|c|c|c|c|c|c|c|}
\hline Stress level & 1 & 2 & 3 & 4 & 5 & 6 & 7 & 8 \\
\hline $\begin{array}{l}\text { Lifetime } \\
\text { logarithm }\end{array}$ & 5.7035 & 5.5660 & 5.6635 & 5.4778 & 5.4032 & 5.3161 & 5.2016 & 5.1120 \\
\hline $\begin{array}{c}\text { Enhancement } \\
\text { coefficient }\end{array}$ & 1 & 1.1593 & 1.0516 & 1.2662 & 1.3643 & 1.4884 & 1.6690 & 1.8255 \\
\hline Stress level & 9 & 10 & 11 & 12 & 13 & 14 & 15 & 16 \\
\hline $\begin{array}{l}\text { Lifetime } \\
\text { logarithm }\end{array}$ & 5.0747 & 4.8589 & 4.9222 & 4.6680 & 4.6323 & 4.5552 & 4.4379 & 4.3263 \\
\hline $\begin{array}{c}\text { Enhancement } \\
\text { coefficient }\end{array}$ & 1.8948 & 2.3512 & 2.2070 & 2.8458 & 2.9492 & 3.1856 & 3.5820 & 4.0050 \\
\hline Stress level & 17 & 18 & 19 & 20 & 21 & 22 & 23 & 24 \\
\hline $\begin{array}{l}\text { Lifetime } \\
\text { logarithm }\end{array}$ & 4.2382 & 4.1410 & 4.0101 & 3.9469 & 3.9733 & 3.7492 & 3.6621 & 3.4614 \\
\hline $\begin{array}{c}\text { Enhancement } \\
\text { coefficient }\end{array}$ & 4.3738 & 4.8203 & 5.4944 & 5.8529 & 5.7004 & 7.1323 & 7.7879 & 9.5108 \\
\hline
\end{tabular}

In summary, it can be concluded that by increasing the speed of the driving sprocket, the purpose of indirectly increasing the positive pressure and the relative sliding speed between the pin shaft and the pin hole is to accelerate the wear of the component surface and shorten the life of the component. This technology of RET has very good effect and can provide reference for engineering practice.

For a product that requires a life test, under the environmental stress of normal work, not only the requirements for the test equipment is high, the test period is long and the cost is high, but under the stress-strength, the service life of the product can be measured in a short time. This greatly shortens the test time, saves costs, and shortens the product's delivery cycle.

\section{Conclusion}

This article explores the RET about wear failure of the reciprocating push-pull chain pin shaft and pin hole fitting of a feeding mechanism. Analyzed its failure mechanism, and determined the technical approach to RET based on virtual prototypes. A feeding mechanism's virtual prototype 
simulation platform was established by simulation software ADAMS. The collision load spectrum and the relative sliding velocity spectrum of the pin and pin hole in the normal working condition and enhanced working condition were obtained, and the single wear amount and enhanced coefficient of the component were obtained. The test shows that this method of enhanced testing is feasible and can achieve the purpose of shortening the life test cycle, and can guide the actual implementation of RET. The work in this paper provides a useful reference for computer-aided design and analysis (RET-CAE) of reliability enhancement testing.

\section{References}

[1] X.S. Wen, X. Xun and C.H. Zhang: RET Theory and Application.( Beijing Science Press, China (2007), p.35.

[2] P.F. Chen and Z.L. Sun: Research on a method of virtual reliability test for piston rod of compressor, J. Military Journal,Vol. 31 (2010) No.9, p.1235-1240.

[3] W. Grzesik and Z. Zalisz: Friction and wear testing of multiplayer coatings on carbide substrates for dry machining applications, J. Surface and Coating Technology, Vol. 155 (2002), p.37-45.

[4] L. Ben and F.R. Xu: Simulation Calculation of Tooth Surface Wear of Straight Tooth Involute Gear, J. Aviation precision manufacturing technology, Vol 5 (2003) No.39, p.29-32.

[5] L. Ben and F.R. Xu: Simulation Calculation of Tooth Surface Wear of Straight Tooth Involute Gear, J. Aviation precision manufacturing technology, Vol 5 (2003) No.39, p.29-32.

[6] Y.F. Yang, J. Zheng and C.C. Di: Reliability Strengthening Test Technology for Artillery Barrel Lapping Mechanism, J. Fire control and Command control, Vol. 41 (2016) No.7, p.175-178. 